\title{
Evaluation of Muscle Strength and Endurance in Postmenopausal Women: A Cross-sectional Study
}

\author{
Arati V Mahishale ${ }^{1}$, Manali P Kulkarni²
}

\begin{abstract}
Background: There are some evidences which show that postmenopausal women have lower levels of muscle strength and physical function than premenopausal women. Although this has been consistently shown, it is unclear whether the associations found are independent of changes in performance or associated with general aging. Studies have been done to evaluate hand grip and pinch strength in postmenopausal women but there still remain controversies if the strength is spared. As there is dearth of literature about strength evaluation in upper limb, lower limb, and trunk and evaluation of cardiovascular endurance in postmenopausal women. The present study is undertaken to evaluate muscle strength and endurance in postmenopausal women.

Objective: To evaluate muscle strength and cardiovascular endurance in postmenopausal women.

Methods: In a cross-sectional study, 65 postmenopausal females were included. Weight, height, BMI, and waist-hip ratio were measured in the study. Muscle strength was evaluated in a group of quadriceps, hamstrings, gluteal, calf, back, and abdominal muscles using a pressure biofeedback unit. Handgrip strength was measured using handheld dynamometer. Cardiovascular endurance was evaluated using 6-minute walk test and step test.

Results: Muscle strength in upper limb and lower limb muscles was decreased when compared to normative values. With Karl Pearson's correlation test, there is a strong correlation between BMI and lower limb muscle strength in gluteal muscles $(p$-value $=0.001)$, in hamstring muscles ( $p$-value $<0.05$ ) and in handgrip strength ( $p$-value $<0.05$ ) with $64.06 \%$ having weak grip strength. Good cardiovascular endurance was seen in most of the women and there was no significant correlation between age and endurance level.

Conclusion: Postmenopausal women have decreased upper limb and lower limb strength with good cardiovascular endurance. Handgrip strength, gluteal, and hamstring muscle strength were positively correlated with BMI and most of the women presented with central obesity. Keywords: Cardiovascular endurance, Muscle strength, Postmenopausal women, Waist-hip ratio.

Journal of South Asian Federation of Obstetrics and Gynaecology (2021): 10.5005/jp-journals-10006-1905
\end{abstract}

\section{INTRODUCTION}

The word menopause gives a direct explanation of the physiological episode in women where menstruation terminates to occur. ${ }^{1,2}$ It is a transitional period for every woman. The levels of the main "reproductive" hormone estrogen, $17 \beta$-estradiol, fall from the mean values of the cyclic life (100-250 pg/mL) to less than $10 \mathrm{pg} / \mathrm{mL}^{3}$ at the menopausal transition. Thus it loses the ability to saturate receptors and to stimulate target cells. This state leads to a functional dearth of estrogenic activity. ${ }^{1,4}$

Menopause presents with various symptoms inside and outside the body. Symptoms related with this estrogen insufficiency can be during or after the menopause. They are mainly hot flushes, insomnia, vaginal dryness, and night sweats. 5,6 There are other related symptoms and conditions like irregular menstrual bleeding, osteoporosis, arteriosclerosis, dyslipidemia, depressed mood, irritability, headache, forgetfulness, dizziness, deterioration in postural balance, palpitation, dry eyes, dry mouth, reduced skin elasticity, restless legs, and muscle and joint pain. ${ }^{6,7}$ but these symptoms are not necessarily linked to estrogen levels.

Age-related decline in muscle performance is one of the crucial factors in the process of frailty. Decline and impaired mobility are serious threats, especially in elderly women who have lower functional capacity ${ }^{8}$ and longer life span compared with men. Some studies have suggested that a sudden decline in muscle performance coincides with the menopause because of the loss of ovarian steroids, especially estradiol. ${ }^{8}$
1'Department of Obstetrics and Gynecology, KLE Physiotherapy, Belagavi, Karnataka, India

${ }^{2}$ Department of Physiotherapy, Tilak Maharashtra Vidyapeeth, Pune, Maharashtra, India

Corresponding Author:Manali P Kulkarni, Department of Physiotherapy, Tilak Maharashtra Vidyapeeth, Pune, Maharashtra, India, Phone: + 91 8095084373, e-mail: manapkulkarni1194@gmail.com

How to cite this article: Mahishale AV, Kulkarni MP. Evaluation of Muscle Strength and Endurance in Postmenopausal Women: A Crosssectional Study. J South Asian Feder Obst Gynae 2021;13(3):163-166.

Source of support: Nil

Conflict of interest: None

A decline in strength is an inherent process of aging. It has been reported extensively as muscle atrophy. ${ }^{9}$ A specific muscle weakness stated as force per cross sectional area has also been demonstrated. ${ }^{9}$ Women might experience a deterioration in physical function during menopause and the studies have shown that the shift through menopause is correlated with a decline in grip as well as pinch strength. ${ }^{10}$ A normal factor in the regulation of force production has been showed for hand muscles. ${ }^{9}$ It is not known whether leg strength is also compromised as a result of ovarian failure in middle-aged women. Weakening of the quadriceps which is happened to be the main weight-bearing muscle group is concerned with ambulation and balance. If the same muscle shows significant weakness, it may result in the greater number of falls in the postmenopausal period. ${ }^{9}$ 


\section{Methodology}

A cross-sectional study was conducted on 64 subjects through nonprobability sampling design for a period of 3 months. Postmenopausal women with age-group of 40-65 years who are willing to participate, working in various schools, colleges, institutions, and housewives in Belgavi city were included in the study.

Subjects who have undergone a hysterectomy or under hormonal replacement therapy, women suffering from any malignancy or fractures, and subjects with cardiovascular problems were excluded from the study.

\section{Outcome Measures}

1. Pressure biofeedback—Pressure biofeedback is a device which helps to register change in pressure in air-filled pressure cell. The unit consists of a combined gauge/inflation bulb. The same is connected to a pressure cell.

2. Handheld dynamometer-Dynamometer is a device that helps to record the force of a muscular contraction. A small handheld dynamometer is used to record the strength or force of muscular contraction of a patient's hand grip.

3. 6-minute walk test (6MWT) - The 6MWT is easy to perform as well as practical test that has been used to assess cardiovascular endurance. It basically calculates the distance that a patient can walk on a flat, hard surface in a period of predefined 6 minutes time.

4. Young Men's Christian Association (YMCA) 3-minute bench step test-3-minute bench step test is constructed on the basis of how quickly your heart rate recovers following a short session of workout.

\section{Procedure}

Ethical consent was obtained from the institutional ethical committee. All participants were screened for inclusion and exclusion criteria. Interested subjects signed the written consent and considered a study subject. The subjects were informed about the aims and procedure of the study. A demographic data was taken and then evaluation of grip strength was done by handheld dynamometer, and lower limb strength was evaluated by pressure biofeedback. Cardiovascular endurance was evaluated by 6MWT and YMCA step bench test.

\section{Results}

The present study aimed to evaluate muscle strength and endurance in postmenopausal women where 65 postmenopausal females were included. Muscle strength was evaluated in quadriceps, hamstrings, gluteal, calf, back, and abdominal muscles using a pressure biofeedback unit. Handgrip strength was measured using a handheld dynamometer. Cardiovascular endurance was evaluated using 6MWT and YMCA bench test.

Statistical analysis was done manually as well as using statistical package of social sciences (SPSS) version 21 for the present study so as to verify the results achieved. Various statistical processes, such as mean and standard deviations, were used. Data from patient's demographic data, i.e., the age, height, weight, BMI, waist-hip ratio, were analyzed using mean and SD. Mean value for age, height, weight, BMI, waist-hip ratio is given in Table 1.
Mean strength for gluteal, hamstrings, quadriceps, calf, abdominals, and back is presented in Table 2 .

The Karl Pearson's correlation test was used to check the association among BMI and lower limb muscle strength and we found highly significant correlation between BMI and muscle strength in gluteal with the $p$-value 0.001 for both the sides and it is significant in hamstring muscles with the $p$-value 0.0496 and 0.0523 for right and left sides, respectively, which is $<0.005$.

According to the normative values of grip strength, $64.06 \%$ of females had weak grip strength and $35.94 \%$ of females had normal grip strength. Karl Pearson's correlation test was used to check the correlation between BMI and grip strength and there is positive between grip strength and BMI with the $p$-value 0.0141 and 0.0119 for right and left sides, respectively, Table 3.

Endurance level was assessed after step test according to norms of YMCA bench test. Heart rate was measured after step test and they were categorized according to cardiovascular endurance level under excellent, good, above average, average, below average, poor, very poor. Cardiovascular endurance was excellent in 20 women (31.25\%), good in 41 women (64.06\%) and above average

Table 1: Mean values of demographic data

\begin{tabular}{lcl}
\hline Demographic data & Mean value & SD \\
\hline Age & 51.70 & 6.007 \\
Height & 1.53 & 0.07 \\
Weight & 57.44 & 9.40 \\
BMI & 23.94 & 4.31 \\
Waist-hip ratio & 0.83 & 0.07 \\
\hline
\end{tabular}

Table 2: Mean strength for a group of muscles

\begin{tabular}{lll}
\hline Muscle group & Right & Left \\
\hline Gluteal & $67.78 \pm 3.26$ & $68.15 \pm 3.19$ \\
Hamstrings & $77.95 \pm 3.01$ & $77.78 \pm 3.21$ \\
Quadriceps & $82.75 \pm 3.05$ & $82.78 \pm 4.05$ \\
Calf & $74.15 \pm 2.99$ & $73.23 \pm 3.19$ \\
Grip & $15.39 \pm 5.47$ & $15.10 \pm 5.35$ \\
Abdominals & $65.37 \pm 4.96$ & \\
Back & $38.78 \pm 2.02$ & \\
\hline
\end{tabular}

Table 3: Correlation between BMI with lower limb strength by Karl Pearson's correlation

\begin{tabular}{lcccc}
\hline & & \multicolumn{3}{c}{ Correlation between BMI with- } \\
\cline { 3 - 5 } Gluteal & Rt & 0.9999 & 18.5200 & $0.0001^{*}$ \\
& Lt & 0.9125 & 17.5631 & $0.0001^{*}$ \\
Hams & Rt & 0.2465 & 2.0030 & $0.0496^{*}$ \\
\multirow{3}{*}{ Quads } & Lt & 0.2344 & 1.8986 & $0.0523^{*}$ \\
& Rt & 0.0442 & 0.3481 & 0.7289 \\
Calf & Lt & -0.0214 & -0.1688 & 0.8665 \\
& Rt & -0.0723 & -0.5709 & 0.5701 \\
Handgrip & Lt & 0.0096 & 0.0754 & 0.9401 \\
& Rt & 0.3056 & 2.5272 & $0.0141^{*}$ \\
& Lt & 0.3125 & 2.5903 & $0.0119^{*}$ \\
\hline
\end{tabular}

${ }^{*} p<0.05$ 
in 3 women (4.69\%). 6MWT was performed on the women and the mean distance covered was $146.40 \pm 12.20$. The results showed that there is no correlation between age and endurance level in postmenopausal women.

\section{Discussion}

In the present study, evaluation of muscle strength and endurance was done in postmenopausal women and the study included 65 postmenopausal females. Muscle strength was evaluated in quadriceps, hamstrings, gluteal, calf, back, and abdominal muscle using a pressure biofeedback unit. Handgrip strength was measured using a handheld dynamometer. Cardiovascular endurance was evaluated using 6MWT and step test.

The present study has evaluated anthropometric measures such as waist-hip ratio, grip strength, lower limb strength, and trunk strength with cardiovascular endurance unlike evaluating individual parameters in other studies. The present study was carried out in an Indian scenario, as there is less literature available in Indian postmenopausal women about strength and endurance as well as the anthropometric values. The results are in consensus with other populations while subjects had good activity level.

The age-group included in this study was $40-65$ years and the mean age was 51.70 years.

According to a study done by Donato, central obesity was defined as a waist to hip ratio is equal to or more than 0.80 for women. In the present study, mean waist-hip ratio was $0.83 \pm 0.07$ in postmenopausal women. It suggests in postmenopausal women central obesity was significant and positively correlated with the above-mentioned study where they found a relation between menopausal status and central adiposity measured at various cutoffs of waist circumference and waist to hip ratio and they established that postmenopausal women are at greater risk of central obesity as evaluated by waist circumference as well as waist to hip ratio. ${ }^{11}$

Mean grip strength in the presented study for the right hand was $15.39 \pm 5.47$ and for left hand was $15.10 \pm 5.35$ and the grip strength values according to age of the women given by JAMAR dynamometer 41 women had weak grip strength and 23 women had normal grip strength. Grip strength was measured as an indicator of muscular strength in upper limbs. In the present study, maximum women showed weak grip strength and it can be due to the advancing age and reduction in physical activity. One more important factor that contributes to decline in grip strength can be loss of estrogen in menopause and positively correlated with a study done by Kurina which concluded that women who entered the postmenopausal period showed a decline in grip strength. ${ }^{12}$ In the present study, we found a positive correlation between $\mathrm{BMI}$ and grip strength as the women with higher BMI have more grip strength and it is positively correlated with a study done by Marsh et al. where they concluded that grip strength and BMI were positively correlated. ${ }^{13}$ A study done by Liao on handgrip strength in individuals with different anthropometric measurements with low, medium, and high body mass indexes of males and females. The study concluded that personal variables like weight, height, and BMI have a positive association with handgrip strength. The study also suggests that high BMI group had a stronger handgrip strength compared to low group as well as handgrip strength was closely associated with BMI. ${ }^{14}$

In the present research, we also evaluated lower limb muscle strength by evaluating strength in gluteal, hamstrings, quadriceps, and calf muscle and the strength in abdominal and back muscle group and it was found to be less than the normative values. It is a proven fact that aging is associated with a reduction in muscle bulk called Sarcopenia. ${ }^{15}$ Sarcopenia can be clinically explained as the mean appendicular muscle mass less by minimum of two standard deviations than young healthy adults of reference populations. Muscle mass decreases gradually after the third decade of age in women and shows significant reduction of muscle mass after the fifth decade. The study done by Tanko et al. ${ }^{16}$ established that muscle mass is correlated in contrary with age and it remains challenging to prove the relative contribution of menopausal transition as opposed to age with regards to loss of muscle mass. The loss of muscle mass is influenced by many contributing factors one of which can be low physical activity. ${ }^{17,18}$ The present study also indicates the positive correlation within BMI and muscle strength specifically in the gluteal as well as hamstrings group of muscles.

Previous studies put forward that when BMI increases, there is a significant increase in muscle mass but it is also proved that muscle mass did not increase in female diabetes patients with more than $25 \mathrm{~kg} / \mathrm{m}^{2} \mathrm{BMI}^{19}$ In present study, mean BMI was $23.94 \pm 4.31$ which may can suggest that there was a positive correlation between $\mathrm{BMI}$ and muscle mass and intern increase in muscle strength.

In this study, we evaluated cardiovascular endurance using step test and age-adjusted standards which were based on YMCA-published guidelines. The cardiovascular endurance of 20 women was excellent and 41 women had good cardiovascular endurance and it suggests that most of the women have adequate cardiovascular endurance and the possible reasons behind it can be the women enrolled in the present were nonsedentary and the amount of activities they do in house or at work daily causes physical exercise and it helps them to maintain physical fitness. According to the study done by Asikainen on postmenopausal women, women who pass the menopausal phase face many latent changes that may lead to deterioration in health-linked fitness especially if they are inactive. ${ }^{20}$ According to the study done by Church et al., there is a positive correlation between the amount of exercise and change in fitness. ${ }^{21}$ Suggestive of the fitness level was good in postmenopausal women in the presented study due to nonsedentary lifestyle and daily activity level.

\section{Conclusion}

Postmenopausal women have decreased upper limb and lower limb strength with good cardiovascular endurance. Handgrip strength, gluteal, and hamstring muscle strength were positively correlated with $\mathrm{BMI}$ and most of the women presented with central obesity.

\section{References}

1. Kakkar V, Kaur D, Chopra K, et al. Assessment of the variation in menopausal symptoms with age, education and working/nonworking status in north-Indian sub population using menopause rating scale (MRS). Maturitas 2007;57(3):306-314. DOI: 10.1016/j. maturitas.2007.02.026.

2. Milson I. Menopause related symptoms and their treatment-the menopause, Chapter 2.

3. Siddiqui NI, Rahman S, Mia AR, et al. Evaluation of hormone replacement therapy. Mymensingh Med J 2005;14(2):212-218 .

4. Burger HG, Dudley EC, Hopper JL, et al. Prospectively measured levels of serum follicle-stimulating hormone, estradiol, and the dimeric inhibins during the menopausal transition in a population-based 
cohort of women 1. J Clin Endocrinol Metab 1999;84(11):4025-4030. DOI: 10.1210/jcem.84.11.6158.

5. Campbell S, Whitehead M. Estrogen therapy and menopausal syndrome Clin Obstet Gynaecol 19774(1):31-47.

6. Oldenhave A, Jaszmann LJ, Haspels AA, et al. Impact of climacteric on well-being: a survey based on 5213 women $39-60$ years old. Am J Obstet Gynecol 1993;168(3):772-780. DOI: 10.1016/s00029378(12)90817-0.

7. Hunter MS. Psychological and somatic experience of the menopause: a prospective study [corrected]. Psychosom Med 1990;52(3):357-367. DOI: 10.1097/00006842-199005000-00009.

8. Sipilä S, Taaffe DR, Cheng S, et al. Effects of hormone replacement therapy and high-impact physical exercise on skeletal muscle in post-menopausal women: a randomized placebo-controlled study. Clin Sci 2001;101(2):147-157.

9. Greeves JP, Nigel T, Reilly T, et al. Changes in muscle strength in women following the menopause: a longitudinal assessment of the efficacy of hormone replacement therapy. Clin Sci 1999;97(1):79-84.

10. Kurina LM, Gulati M, Everson-Rose SA, et al. The effect of menopause on grip and pinch strength: results from the Chicago, Illinois, site of the study of Women's Health Across the Nation. Am J Epidemiol 2004;160(5):484-491. DOI: 10.1093/aje/kwh244.

11. Donato GB, Fuchs SC, Oppermann K, et al. Association between menopause status and central adiposity measured at different cutoffs of waist circumference and waist-to-hip ratio. Menopause 2006;13(2):280-285.

12. Phillips SK, Rook KM, Siddle NC, et al. Muscle weakness in women occurs at an earlier age than in men, but strength is pre-served by hormone replacement therapy. Clin Sci (Lond) 1993;84:95-98.
13. Marsh AP, Rejeski WJ, Espeland MA, et al. Muscle strength and BMI as predictors of major mobility disability in the Lifestyle Interventions and Independence for Elders Pilot (Life-P). J Gerontol Ser A 2011;66(12):1376-1383. DOI: 10.1093/gerona/glr158.

14. Liao KH. Hand grip strength in low, medium, and high body mass index males and females. Middle East J Rehabil Health 2016;3(1). DOI: 10.17795/mejrh-33860.

15. Morley JE, Baumgartner RN, Roubenoff R, et al. Sarcopenia. J Lab Club Med 2001;137(4):231-243. DOI: 10.1067/mlc.2001.113504.

16. Movsesyan L, Mouritzen U, Christiansen C, et al. Appendicular lean tissue mass and the prevalence of sarcopenia among healthy women. Metabolism 2002;51(1):69-74. DOI: 10.1053/meta.2002.28960.

17. Aloia JF, Mcgowan DM, Vaswani AN, et al. Relationship of menopause to skeletal and muscle mass. Am J Clin Nutr 1991;53(6):1378-1383. DOI: $10.1093 / a j c n / 53.6 .1378$.

18. Hansen RD, Allen BJ. Habitual physical activity, anabolic hormones, and potassium content of fat-free mass in postmenopausal women. Am J Clin Nutr 2002;75(2):314-320. DOI: 10.1093/ajcn/75.2.314.

19. Kwon $\mathrm{HR}$, Han $\mathrm{KA}$, Ku YH, et al. Relationship of maximal muscle strength with body mass index and aerobics capacity in type 2 diabetic patients. Korean Diab J 2009;33(6):511-517. DOI: 10.4093/kdj.2009.33.6.511.

20. Asikainen TM, Kukkonen-Harjula K, Miilunpalo S. Exercise for health for early postmenopausal women: a systematic review of randomised controlled trials. Sports Med 2004;34(11):753-779. DOI: 10.2165/00007256-200434110-00004.

21. Church TS, Martin CK, Thompson AM, et al. Changes in weight, waist circumference and compensatory responses with different doses of exercise among sedentary, overweight postmenopausal women. PLoS One 2009;4(2):E4515. DOI: 10.1371/journal.pone.0004515. 